\title{
Variabilidad de la demanda del tiempo de entrega, existencias de seguridad y costo del inventario
}

\author{
Lead time demand variability, safety stock and the inventory cost \\ Juan Manuel Izar Landeta ${ }^{\mathrm{a}, *}$, Carmen Berenice Ynzunza Cortés ${ }^{\mathrm{b}}$ \\ y Orlando Guarneros García ${ }^{a}$ \\ ${ }^{a}$ Universidad Autónoma de San Luis Potosí, México \\ ${ }^{\mathrm{b}}$ Universidad Tecnológica de Querétaro, México
}

Recibido el 19 de noviembre de 2014; aceptado el 10 de abril de 2015

Disponible en Internet el 27 de marzo de 2016

\section{Resumen}

Este trabajo tiene como objetivo analizar los efectos que la variabilidad de la demanda del tiempo de entrega tiene sobre las existencias de seguridad y el costo del inventario de artículos. El análisis se aplica a 2 productos para los cuales se tiene información, uno con distribución normal de la demanda del tiempo de entrega y el otro con distribución uniforme. Los costos analizados son el de mantenimiento del inventario de seguridad y el de los faltantes que hay cuando la demanda de los consumidores supera las existencias. El inventario de seguridad se establece en aquel valor que minimice la suma de los 2 costos antes comentados, luego se analiza cómo afecta a este valor la variabilidad de la demanda. Los resultados han sido similares para los 2 productos analizados. Con más variabilidad de la demanda, las existencias de seguridad aumentan de manera lineal, sin que se modifique este comportamiento en caso de variar la estructura de los costos de mantenimiento y faltantes. Asimismo, con más variabilidad de la demanda el costo mínimo local aumenta, en mayor grado para el artículo con distribución normal. El nivel de servicio de costo mínimo se localiza en un valor por debajo del 100\% para ambos artículos: 94.5\% para el de distribución normal y 96\% para el de

\footnotetext{
* Autor para correspondencia.

Correo electrónico: jmizar@uaslp.mx (J.M. Izar Landeta).

La revisión por pares es responsabilidad de la Universidad Nacional Autónoma de México.
} 
distribución uniforme. Para los 2 artículos se ha dado una relación inversa entre el nivel de servicio de costo mínimo y la razón del costo de mantenimiento y faltantes.

Derechos Reservados (C) 2016 Universidad Nacional Autónoma de México, Facultad de Contaduría y Administración. Este es un artículo de acceso abierto distribuido bajo los términos de la Licencia Creative Commons CC BY-NC-ND 4.0.

\title{
Códigos JEL: L11; L23
}

Palabras clave: Inventario de seguridad; Demanda del tiempo de entrega; Variabilidad de la demanda; Nivel de servicio; Costos del inventario

\begin{abstract}
The objective of this research is to investigate the impacts of the lead-time demand variability on the safety stock and the inventory cost.

The analysis is applied to two products for which data is available, one with a normal distribution of lead time demand and the other with uniform distribution. The costs analyzed are the safety stock maintenance and the shortages when the consumer demand exceeds availability.

The safety stock is set at the value that minimizes the sum of the two previously mentioned costs and is then analyzed how the demand variability affects this stock.

The results were similar for the two products tested. With more demand variability, safety stock increases linearly; this behavior is maintained, even if the cost structure of maintenance or shortages are modified. Likewise, with more demand variability the minimum cost increases to a greater extent for the item with a normal distribution. The service level of the minimum cost is located at a value below $100 \%$ for both items, $94.5 \%$ for the normal distribution item and $96 \%$ for the uniform distribution item.

In summary, the two articles exhibited an inverse relationship between the service level of the minimum cost and the ratio of the maintenance cost and the shortages.

All Rights Reserved () 2016 Universidad Nacional Autónoma de México, Facultad de Contaduría y Administración. This is an open access item distributed under the Creative Commons CC License BY-NC-ND 4.0.
\end{abstract}

JEL classification: L11; L23

Keywords: Safety stock; Lead time demand; Demand variability; Service level; Inventory costs

\section{Introducción}

En el contexto de la administración de inventarios, es bien sabido que ante la variación de la demanda y la demora en la entrega de un nuevo pedido por parte del proveedor las organizaciones definen un inventario de seguridad que les permita atender la demanda y no llegar a la penosa situación de tener agotamientos del inventario que les representarían ventas perdidas, así como una mala imagen ante los consumidores, lo cual hoy día es esencial ante la enorme competencia que se ha desatado en prácticamente todos los sectores comerciales.

Chikan (2007) señala que hay un cambio de paradigma respecto al manejo de los inventarios, que las organizaciones actuales deben entender, de modo que no se convierta en una desventaja competitiva. Antes, el manejo del inventario se fundamentaba en 3 pilares: 1) era independiente de otras cuestiones administrativas; 2) su función principal era amortiguar las diferencias entre la oferta y una demanda incierta, y 3) la medida de desempeño era el costo del inventario que incluía el mantenimiento, hacer nuevos pedidos y la eventual aparición de faltantes. El nuevo paradigma que propone el autor es que los inventarios sean parte integral de la cadena de valor, estrechamente relacionados con otras funciones de la organización, convirtiéndose en una 
herramienta estratégica que logre beneficios económicos y la satisfacción del cliente, y las medidas de desempeño deben basarse en encontrar mejores soluciones para el consumidor que las que le ofrecen los competidores.

Barry (2007) sugiere como métricas para el manejo del inventario las tasas inicial y final de órdenes atendidas, la tasas de artículos devueltos y cancelaciones, la rotación del inventario y el margen de retorno de la inversión.

Este trabajo analiza el impacto que sobre las existencias de seguridad y el costo del inventario tiene la variabilidad de la demanda del tiempo de entrega de 2 artículos para los cuales se tiene información relativa al inventario, ambos con demanda independiente, uno con distribución normal y el otro uniforme, bajo un sistema de revisión periódica. Para estimar las existencias de seguridad, estas se determinan en un valor que minimice el costo del inventario, tomando en cuenta solo los costos de faltantes, que se estiman como lo que se deja de ganar por no disponer de los artículos cuando los pide el consumidor, así como el costo de mantenimiento de dicho inventario. Por lo tanto, no se toman en cuenta los costos de hacer nuevos pedidos, ni el de adquisición de los productos. Asimismo se busca explicar la relación del nivel de servicio con el costo del inventario y la estructura de costos.

\section{Fundamentos teóricos}

En el ámbito de los inventarios se han efectuado numerosos estudios de aplicaciones de varios modelos a casos prácticos para determinar las existencias de seguridad, buscando la mayoría de ellos minimizar el costo implicado.

Para este trabajo se ha realizado una revisión de la literatura, en particular de aquella que tiene que ver con la determinación del inventario de seguridad en el caso de manejar tiempos de entrega aleatorios, para el caso de artículos que no se manufacturan en la empresa, sino que se compran a un proveedor para su venta posterior al consumidor y que es el caso típico de muchas Pymes mexicanas que enfrentan en esta época serios problemas de supervivencia.

En las cadenas de suministro se buscan mecanismos para reducir el nivel de inventario sin menoscabo del nivel de servicio provisto al cliente. Chopra, Reinhardt y Dada señalan que 2 opciones para conseguir esto son la reducción del tiempo de entrega del proveedor y de la variabilidad de este tiempo de entrega. Si la demanda del tiempo de entrega sigue una distribución normal, estas 2 acciones reducen el inventario para niveles de servicio superiores al $50 \%$ y es de mayor impacto la variabilidad del tiempo de entrega que su magnitud.

Silver (1965) comenta que la manera tradicional de calcular el punto de reorden es para un nivel de servicio meta, la cual asume que la demanda del tiempo de entrega se ajusta a una distribución de probabilidad conocida, lo que muchas veces no es válido, particularmente en el caso de un artículo que se mueve de manera lenta y del cual se dispone de pocos registros. Para ello propone un proceso bayesiano para calcular el punto de reorden.

Por su parte, Silver y Costa (1995) proponen que las existencias de seguridad se calculen con la mayor demanda del artículo que se tenga registrada, multiplicada por el tiempo de entrega.

Balakrishnan, Pangburn y Stavrulaki (2004) afirman que en algunos casos mantener grandes cantidades de inventario mejora los niveles de servicio y estimula la demanda de artículos. Muestran en su investigación que el ciclo óptimo del inventario depende del balance entre los costos de colocar pedidos y mantener el inventario, mientras que el punto de reorden se define con un análisis costo-beneficio.

Fiom (2012) señala que el inventario de seguridad es una protección contra la incertidumbre, que puede incluir variaciones en la demanda de los clientes, del tiempo de entrega del proveedor y 
de la calidad de los productos que se suministran al cliente.Van Kampen, van Donk y van-der Zee (2010) afirman que las 2 medidas usuales para enfrentar la incertidumbre de la oferta y la demanda de un artículo son las existencias de seguridad y la seguridad del tiempo de entrega. Sin embargo, estos fenómenos se han estudiado en condiciones aisladas, pasando por alto las incertezas del lado de la oferta y la demanda. Han estudiado los efectos del inventario de seguridad y la seguridad del tiempo de entrega en un sistema multi-producto mediante simulación y encuentran que, ante la incertidumbre del lado de la oferta, es mejor manejar un tiempo de entrega con margen de seguridad, y en caso de que la incertidumbre esté del lado de la demanda, es preferible manejar un inventario de seguridad adecuado. En contraste a estudios previos para un único artículo, si hay desconfianza de la información de la oferta y demanda de un único producto, sugieren que la mejor medida para la correcta administración del inventario es la el manejo de un tiempo de entrega seguro.Kanet, Gorman y Stoblëin (2010) sugieren el uso de un inventario de seguridad variable para ahorrar en su administración, con un valor mayor si la demanda o la oferta son inciertas y un menor inventario de seguridad cuando haya mayor certeza en la oferta y/o demanda.

Boute, Disney, Lambrecht y van Houdt (2010) han estudiado el caso de una cadena de suministro de 2 escalones en la cual un comerciante al menudeo se provee de un productor que tiene un tiempo de entrega dado y le sirve a un cliente cuya demanda es sensible a promociones de precios y mercadeo, lo que resulta en una demanda autocorrelacionada. Con una variabilidad igual, si la demanda se autocorrelaciona negativamente, se producen tiempos de entrega del productor más cortos y, por tanto, el inventario de seguridad resulta más pequeño comparado con el caso en que la demanda se autocorrelacione positivamente.

King (2011) comenta que el manejo del inventario busca cumplir con 2 metas fundamentales: dar el nivel de servicio deseado por el cliente y que se haga a un costo mínimo del inventario, que son objetivos que deben balancearse para encontrar una solución apropiada. Afirma que es la variabilidad de la demanda del tiempo de entrega la variable de mayor influencia en esta decisión.

Buzacott y Shanthikumar (1994) señalan que tanto el tiempo de entrega como las existencias de seguridad son los 2 parámetros que determinan el punto de reorden, y ambos dependen de la precisión de los pronósticos con los que se obtienen. Afirman que de los 2 es más importante el tiempo de entrega. Señalan que es preferible un tiempo seguro de entrega en caso que sea posible hacer pronósticos precisos de la demanda futura; de otro modo, será mejor mantener un inventario de seguridad para manejar cambios en los requerimientos del cliente durante el tiempo de entrega, o si hay fluctuaciones en los pronósticos de la demanda.Babai, Syntetos, Dallery y Nikolopoulos (2009) se enfocan en un sistema de inventarios de artículo único con demanda no estacional y tiempo de entrega incierto. Analizan una política de control dinámico del punto de reorden en la cual determinan sus parámetros en función del nivel de servicio meta. Usan un conjunto de datos de la industria farmacéutica. Su estudio tiene 3 ventajas: 1) sus resultados son generales, ya que no se aplican solo para una distribución dada de la demanda; 2) tienen en cuenta la incertidumbre del tiempo de entrega, y 3) no son intensivos desde el punto de vista computacional. La política de control dinámico del punto de reorden tiene un desempeño similar al de la política estática, en términos del nivel de servicio logrado, pero con menores costos del inventario.

Ben-Daya y Hariga (2003) consideran el tiempo de entrega como una variable controlable, la que descomponen en 3 partes: el tiempo de preparación, el tiempo de procesamiento y el tiempo no productivo. Para un buen manejo del tiempo de entrega debe buscarse la reducción del tiempo de preparación y la adecuada interacción entre el tamaño del lote y el tiempo de entrega.

Wang y Hill (2006) encuentran que si el tiempo de entrega sigue una distribución gamma, para niveles de servicio entre el 60 y 70\%, la disminución de la variabilidad del tiempo de entrega primero incrementa el inventario de seguridad y luego lo hace disminuir o mantenerse 
constante, siendo un efecto recursivo, contrario a lo que se ha encontrado con otras distribuciones de probabilidad.

Nasri, Affisco y Paknejad (1990) plantean, ante tiempos de entrega estocásticos con distribuciones de probabilidad normal y uniforme, reducir los costos de hacer pedidos mediante la inversión para disminuir los tiempos de preparación de los pedidos, lo que hace disminuir el tamaño del pedido y proporciona flexibilidad. Concluyen que la reducción de los costos de colocación de pedidos y de la variabilidad del tiempo de entrega son medidas que deben buscarse conjuntamente.

En un estudio para un solo artículo con demanda independiente bajo un proceso de Poisson compuesto con tiempo de entrega estocástico, siendo la medida de desempeño el costo del inventario a largo plazo, Song (1994) encuentra que si el tiempo de entrega se incrementa, esto trae consigo un aumento de la demanda del tiempo de entrega y un mayor inventario de seguridad, aunque no necesariamente un costo óptimo del inventario mayor. Si el tiempo de entrega tiene más variabilidad (mayor desviación estándar), lleva a una mayor demanda del tiempo de entrega y un costo óptimo del inventario más alto. El efecto de la variabilidad depende de la estructura de costos del inventario, concretamente de la razón del costo de faltantes al de la suma de faltantes y mantenimiento del inventario.

Eppen y Martin (1988) sugieren un procedimiento para calcular correctamente las existencias de seguridad en el caso de tiempos de entrega y demandas estocásticas, mostrando que bajo el supuesto de normalidad ocurren errores en su cálculo, los que pueden corregirse siguiendo un algoritmo propuesto, el que aplica en caso que los parámetros de la demanda y el tiempo de entrega sean conocidos o desconocidos.

Ruiz-Torres y Mahmoodi (2010) han propuesto, a partir del modelo de Estes, un nuevo procedimiento basado en datos históricos de la demanda y el tiempo de entrega para calcular las existencias de seguridad, que no asume ninguna distribución de probabilidad y produce resultados más próximos al nivel de servicio meta y menores costos del inventario que los modelos tradicionales, lo que confirma que estos modelos sobreestiman el punto de reorden.

Wang, Zinn y Croxton (2010) han derivado fórmulas para estimar el punto de reorden y las existencias de seguridad en caso de que la demanda y el tiempo de entrega estén correlacionados, ya que los modelos tradicionales asumen que estas 2 variables no están correlacionadas, lo cual puede que no sea así, como en el caso que un cliente importante haga pedidos de mayor volumen para satisfacer la demanda, o en caso que la demanda vaya a la baja y, en consecuencia, el proveedor haga corridas de producción más pequeñas y se tarde más en su entrega.

Dullaert y Zamparini (2013), mediante simulación, muestran que la reducción de la variabilidad del tiempo de entrega no necesariamente disminuye los costos del inventario de seguridad, ya que podría incluso incrementarlo, lo cual depende de la distribución de la demanda del tiempo de entrega y del nivel de servicio meta.

Hayya, Harrison y Chatfield (2009) plantean el cuestionamiento si es posible desarrollar una relación del costo del inventario en función de la desviación estándar de la demanda del tiempo de entrega, llegando a la conclusión que es posible obtener expresiones aproximadas de tal relación, las que apuntan a que si se desea reducir el costo del inventario, la desviación estándar del tiempo de entrega debe disminuirse.

He, Kim y Hayya (2005) establecen que con una tasa de demanda constante es la varianza, y no la media del tiempo de entrega, lo que afecta al costo relevante del inventario en un modelo estocástico del tiempo de entrega.

Fang, Zhang, Robb y Blackburn (2013) afirman que no puede establecerse una conclusión contundente sobre el impacto de reducir la media o la varianza del tiempo de entrega para 
incrementar la rentabilidad del manejo del inventario, ya que esta decisión depende de las condiciones de la demanda y de si estos 2 parámetros están o no correlacionados.

Kouvelis y Li (2008) sugieren el uso de un proveedor de respaldo flexible para satisfacer la demanda del consumidor, lo que es de gran utilidad en caso que la incertidumbre del tiempo de entrega del proveedor usual sea elevada.

\section{Metodología}

Este trabajo calcula para un valor de la desviación estándar de la demanda del tiempo de entrega, el costo del inventario de seguridad, que incluye los faltantes y su mantenimiento. Esto se hace para 2 artículos, uno con distribución de la demanda normal y el otro con demanda uniforme, para los cuales se cuenta con la información necesaria para hacer este análisis, de modo que el aporte de este trabajo es práctico.

El número de faltantes (Nf) cada vez que se hace un pedido se obtiene mediante integración matemática de la función de probabilidad respectiva, conforme a la ecuación siguiente (Taha, 2004):

$$
N f=\int_{P R}^{L S}(x-P R) f(x) d x
$$

Donde:

$\mathrm{X}=$ demanda del tiempo de entrega, unidades.

$\mathrm{PR}=$ punto de reorden, unidades.

LS = límite superior de la distribución de probabilidad, unidades.

$\mathrm{f}(\mathrm{x})=$ función de probabilidad.

Ya que habrá faltantes cada vez que la demanda del tiempo de entrega supere al punto de reorden.

Para el caso de la distribución normal, la ecuación es:

$$
N f=\int_{P R}^{\infty}(x-P R) \frac{\operatorname{Exp}\left[-(x-\mu)^{2} / 2 \sigma^{2}\right]}{\sigma \sqrt{2 \pi}} d x
$$

Donde $\mu$ y $\sigma$ son la media y desviación estándar de la demanda del tiempo de entrega.

Dada la complejidad de esta ecuación, la integral se obtiene numéricamente para encontrar el número de faltantes con cada valor del punto de reorden, o bien con ayuda de un software especializado como Excel, que es el que se ha utilizado en este trabajo.

Para calcular el número de faltantes con Excel, se aplica la siguiente relación:

$$
\mathrm{Nf}=\sigma(\operatorname{DISTR} \cdot \operatorname{NORM}(B / \sigma, 0,1,0))-B(1-\operatorname{DISTR} \cdot \operatorname{NORM}(B / \sigma, 0,1,1))
$$

DISTR.NORM es una función de Excel que facilita el cálculo de los faltantes.

Para el caso del producto con distribución uniforme, la integración se hace fácilmente para llegar a la siguiente ecuación:

$$
N f=\int_{P R}^{a} \frac{x-P R}{a-b} d x=\frac{(a-P R)^{2}}{2(a-b)}
$$


siendo a y b los límites superior e inferior de la distribución uniforme de la demanda del tiempo de entrega.

Para cada valor del punto de reorden se calcula el número de faltantes con la ecuación respectiva, se definen las existencias de seguridad y se calculan los costos de mantenimiento y faltantes, eligiendo el que resulte con el costo mínimo.

El punto de reorden y el costo del inventario se obtienen mediante las ecuaciones (5) y (6), respectivamente:

$$
\begin{aligned}
& P R=\mu+B \\
& C t=C_{\text {man }} B+C_{f a l} N f\left(\frac{D}{Q}\right)
\end{aligned}
$$

Donde:

$\mathrm{Ct}=$ costo total del inventario de seguridad, \$/año.

$\mathrm{C}_{\text {man }}=$ costo de mantener una unidad en inventario, \$/año.

$\mathrm{B}=$ existencias de seguridad, unidades.

$\mathrm{C}_{\text {fal }}=$ costo de cada faltante, $\$ /$ faltante.

$\mathrm{D}=$ demanda anual de artículos, unidades/año.

$\mathrm{Q}=$ cantidad de pedido, unidades/pedido.

En la ecuación (6) el primer término es el costo de mantener el inventario de seguridad y el segundo el de los faltantes, referidos ambos a una base anual.

Para calcular el nivel de servicio, se aplica la fórmula:

$$
z=\frac{P R-\mu}{\sigma}
$$

En la cual Z es el número de desviaciones estandarizadas donde se ubica el punto de reorden y el nivel de servicio es el área correspondiente a este valor de Z para cada distribución de probabilidad.

\section{Aplicación al producto con demanda normal}

Una empresa vende un producto cuya demanda del tiempo de entrega sigue una distribución normal con media 800 y desviación estándar de 150 unidades. Se hacen 28 pedidos anuales, el costo de cada faltante es 60 pesos y el de mantener una unidad en inventario es 45 pesos anuales.

Se busca definir cómo afecta al inventario de seguridad y al costo del inventario cambios en la desviación estándar de la demanda, así como la relación entre el nivel de servicio con el costo del inventario y la razón de los costos de mantenimiento y faltantes.

\section{Resultados y discusión}

Lo primero es calcular para la desviación estándar del caso base $(\sigma=150)$ y cada valor del punto de reorden PR de la media hacia arriba, el número de faltantes y los costos de estos y de mantenimiento del inventario de seguridad. Estos resultados se presentan en la tabla 1.

El mínimo local ha resultado en un punto de reorden de 1,080 unidades, con un inventario de seguridad de 280 y un nivel de servicio de $96.9 \%$, para un costo total por mantenimiento y faltantes de 15,636 pesos. 
Tabla 1

Opciones de valores de PR con sus costos

\begin{tabular}{|c|c|c|c|c|c|c|c|}
\hline PR & $\mathrm{B}$ & $\mathrm{B} / \sigma$ & $\mathrm{Nf}$ & ns & Cman & Cfal & Ctotal \\
\hline 800 & 0 & 0.00 & 59.84 & 0.500 & 0.00 & $100,533.45$ & $100,533.45$ \\
\hline 840 & 40 & 0.27 & 41.96 & 0.605 & $1,800.00$ & $70,486.94$ & $72,286.94$ \\
\hline 880 & 80 & 0.53 & 28.16 & 0.703 & $3,600.00$ & $47,302.03$ & $50,902.03$ \\
\hline 920 & 120 & 0.80 & 18.03 & 0.788 & $5,400.00$ & $30,292.22$ & $35,692.22$ \\
\hline 960 & 160 & 1.07 & 10.99 & 0.857 & $7,200.00$ & $18,462.58$ & $25,662.58$ \\
\hline 1000 & 200 & 1.33 & 6.36 & 0.909 & $9,000.00$ & $10,683.57$ & $19,683.57$ \\
\hline 1040 & 240 & 1.60 & 3.49 & 0.945 & $10,800.00$ & $5,856.98$ & $16,656.98$ \\
\hline 1080 & 280 & 1.87 & 1.81 & 0.969 & $12,600.00$ & $3,036.30$ & $15,636.30$ \\
\hline 1120 & 320 & 2.13 & 0.88 & 0.984 & $14,400.00$ & $1,485.97$ & $15,885.97$ \\
\hline 1160 & 360 & 2.40 & 0.41 & 0.992 & $16,200.00$ & 685.55 & $16,885.55$ \\
\hline 1200 & 400 & 2.67 & 0.18 & 0.996 & $18,000.00$ & 297.77 & $18,297.77$ \\
\hline 1240 & 440 & 2.93 & 0.07 & 0.998 & $19,800.00$ & 121.64 & $19,921.64$ \\
\hline 1280 & 480 & 3.20 & 0.03 & 0.999 & $21,600.00$ & 46.68 & $21,646.68$ \\
\hline 1320 & 520 & 3.47 & 0.01 & 1.000 & $23,400.00$ & 16.82 & $23,416.82$ \\
\hline 1360 & 560 & 3.73 & 0.00 & 1.000 & $25,200.00$ & 5.68 & $25,205.68$ \\
\hline 1400 & 600 & 4.00 & 0.00 & 1.000 & $27,000.00$ & 1.80 & $27,001.80$ \\
\hline
\end{tabular}

Fuente: elaboración propia.

Si se repiten estos cálculos para encontrar la relación de cambio entre $\sigma$ y $\mathrm{B}$, se generan los resultados que se muestran en la figura 1, en la que puede verse que la relación entre $\sigma$ y B es directa y lineal, aumentando las existencias de seguridad 1.88 unidades por cada unidad de incremento de la desviación estándar, lo cual era de esperar. En la figura se ha incluido el ajuste de la relación con su valor de R cuadrada, que señala que ha sido un muy buen ajuste entre las variables.

La figura 2 muestra la relación entre la desviación estándar de la demanda del tiempo de entrega y el costo mínimo local, en la cual se ve que la relación es directa y lineal perfecta $\left(\mathrm{R}^{2}=1\right)$, lo que era de esperar e indica que por cada unidad que aumente la desviación estándar de la demanda, el costo se incrementa 104 pesos.

Si se busca ahora la relación entre el nivel de servicio y el costo del inventario, esta se presenta en la figura 3, para un valor de la desviación estándar del caso base, 150 unidades:

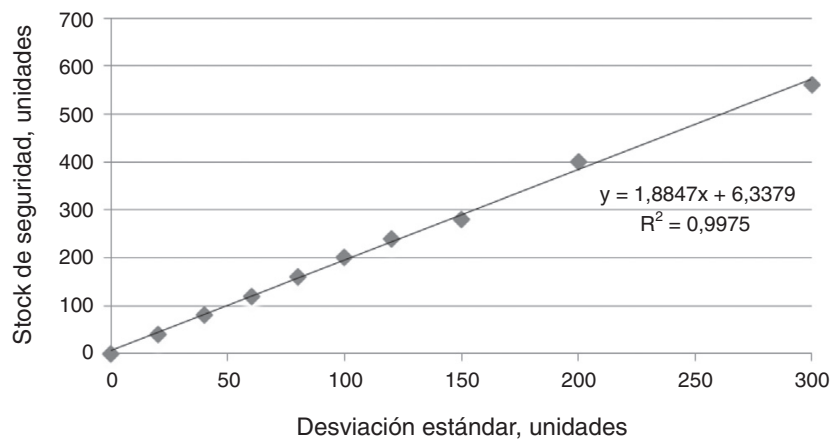

Figura 1. Relación del inventario de seguridad con $\sigma$. Fuente: elaboración propia. 


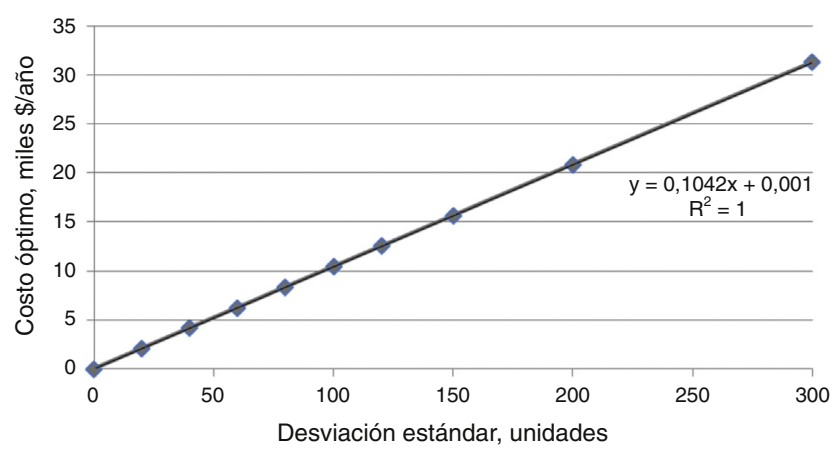

Figura 2. Relación entre $\sigma$ y el costo mínimo local.

Fuente: elaboración propia.

Se observa que el costo mínimo se da para un nivel de servicio de $94.5 \%$, con un monto de 12,510 pesos anuales; no ha sido con el $100 \%$, ya que en este valor el costo incremental de mantener un mayor inventario de seguridad rebasa al ahorro de no tener faltantes. Esta situación del valor el nivel de servicio para el costo mínimo del inventario se repite en caso de variar la desviación estándar del tiempo de entrega.

Si ahora se analiza cómo afecta la estructura de los costos al nivel de servicio de costo mínimo, lo que hay que hacer es variar la razón del costo de mantenimiento respecto al de faltantes, que para el caso base ha sido de 0.75 (45/60). Dicha relación se presenta en la figura 4 y, tal y como se esperaba, la relación es inversa y lineal, ya que si la razón de costos aumenta, es más caro mantener existencias de seguridad y menos caro tener faltantes, con lo cual el nivel de servicio disminuye. El nivel de ajuste de la relación ha sido muy bueno, ya que R cuadrada es 0.96, y por cada unidad que aumente la razón de los costos, el nivel de servicio de costo mínimo disminuye $3.39 \%$.

Además se repitieron estos cálculos modificando la estructura de los costos de faltantes y mantenimiento del inventario, a fin de verificar si estos patrones de variación cambiaban, lo que no sucedió, ya que las relaciones señaladas antes se mantuvieron.

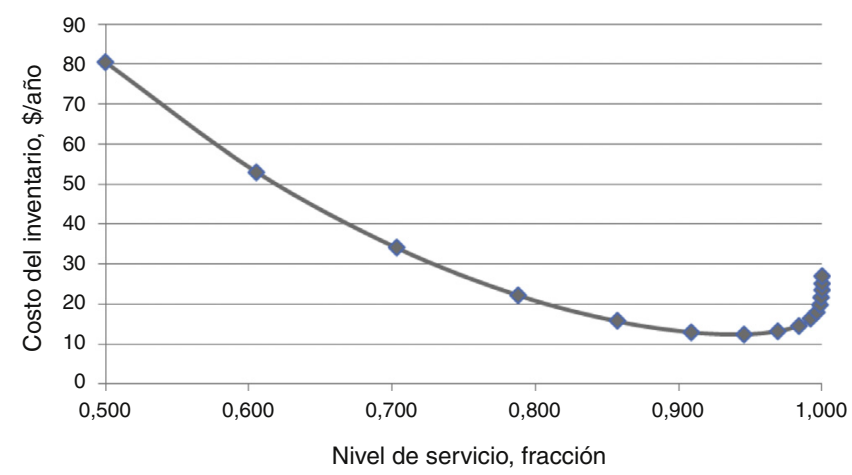

Figura 3. Relación entre el nivel de servicio y el costo del inventario. Fuente: elaboración propia. 


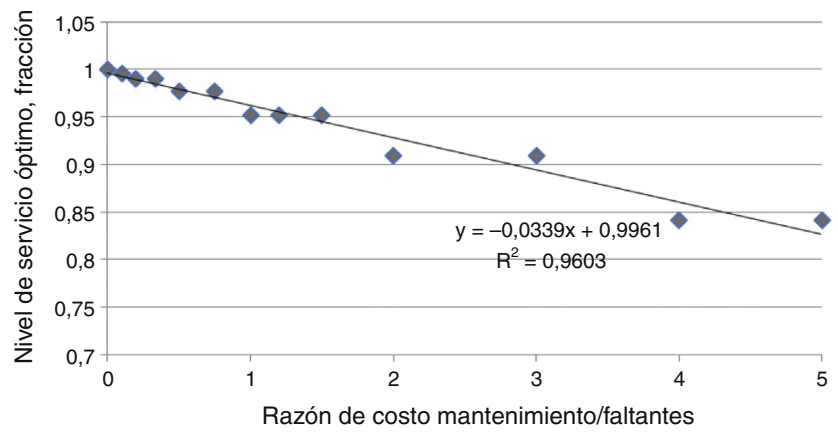

Figura 4. Cambio del nivel de servicio con la razón de costo mantenimiento/faltantes.

Fuente: elaboración propia.

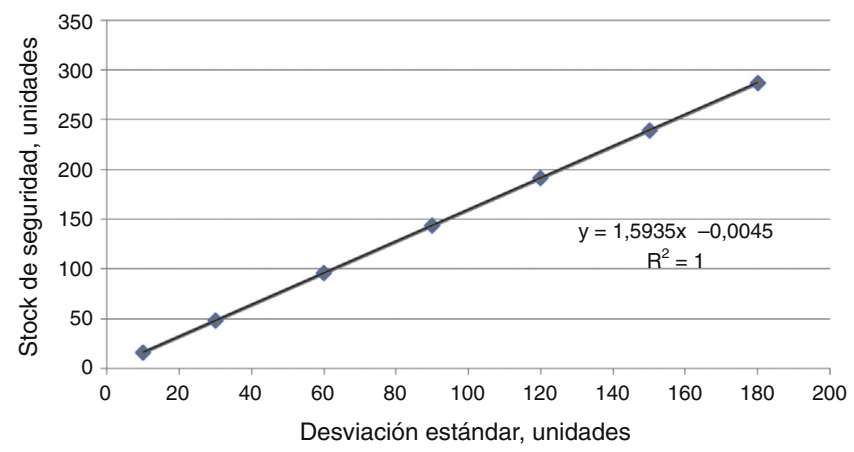

Figura 5. Relación de las existencias de seguridad y $\sigma$.

Fuente: elaboración propia.

\section{Aplicación al producto con demanda uniforme}

Para el artículo con demanda del tiempo de entrega uniforme, su media es de 600 y su desviación estándar de 120 unidades, con un costo por faltante de $\$ 100$ y de mantenimiento de 140 pesos anuales por artículo. El negocio hace 34 pedidos anuales. Se desea conocer el efecto de la desviación estándar sobre las existencias de seguridad y el costo del inventario, así como la relación entre el nivel de servicio y el costo del inventario y la razón de costos de mantenimiento $\mathrm{y}$ faltantes.

\section{Resultados y discusión}

Para los datos del caso base se calcula, para cada nivel de servicio, el punto de reorden, las existencias de seguridad, el número de faltantes y el costo de mantenimiento, faltantes y total, los cuales se presentan en la tabla 2, donde puede verse que el mínimo local ha sucedido para un nivel de servicio del $96 \%$, con un punto de reorden de 791 unidades y un inventario de seguridad de 191 , con un costo total de 27,901 pesos anuales.

Si se hacen estimaciones para encontrar la relación de $\sigma$ con $\mathrm{B}$, se generan los resultados que se muestran gráficamente en la figura 5, en la que puede verse que, al igual que el producto anterior, la relación es directa y lineal, con un ajuste perfecto, medido por el coeficiente de determinación, 
siendo la relación de cambio de 1.59 unidades más en el inventario de seguridad por cada unidad de incremento en la desviación estándar de la demanda.

Para la variación del costo mínimo local con la desviación estándar, esta se muestra en la figura 6.

La relación es directa y lineal, con un ajuste perfecto, incrementándose el costo en 201 pesos por cada unidad de aumento en la desviación estándar de la demanda.

Para el cambio del costo del inventario con el nivel de servicio, la figura 7 presenta esta relación entre ambas variables para el valor de la desviación estándar de 120 unidades, la cual muestra que el nivel de servicio de costo mínimo se da a un valor del $96 \%$, con un costo por un monto de 24,077 pesos anuales, y al igual que en el caso anterior, este valor del nivel de servicio de costo mínimo se repite en caso de variar la desviación estándar.

Tabla 2

Opciones de valores de ns, PR y sus costos

\begin{tabular}{|c|c|c|c|c|c|c|}
\hline ns & PR & B & $\mathrm{Nf}$ & Cman & Cfal & Ctotal \\
\hline 0.50 & 600.00 & 0.00 & 51.96 & 0.00 & $176,669.18$ & $176,669.18$ \\
\hline 0.55 & 620.78 & 20.78 & 42.09 & $2,909.85$ & $143,102.04$ & $146,011.88$ \\
\hline 0.60 & 641.57 & 41.57 & 33.26 & $5,819.69$ & $113,068.28$ & $118,887.97$ \\
\hline 0.65 & 662.35 & 62.35 & 25.46 & $8,729.54$ & $86,567.90$ & $95,297.44$ \\
\hline 0.70 & 683.14 & 83.14 & 18.71 & $11,639.38$ & $63,600.91$ & $75,240.29$ \\
\hline 0.75 & 703.92 & 103.92 & 12.99 & $14,549.23$ & $44,167.30$ & $58,716.52$ \\
\hline 0.80 & 724.71 & 124.71 & 8.31 & $17,459.07$ & $28,267.07$ & $45,726.14$ \\
\hline 0.85 & 745.49 & 145.49 & 4.68 & $20,368.92$ & $15,900.23$ & $36,269.14$ \\
\hline 0.90 & 766.28 & 166.28 & 2.08 & $23,278.76$ & $7,066.77$ & $30,345.53$ \\
\hline 0.92 & 774.59 & 174.59 & 1.33 & $24,442.70$ & $4,522.73$ & $28,965.43$ \\
\hline 0.95 & 787.06 & 187.06 & 0.52 & $26,188.61$ & $1,766.69$ & $27,955.30$ \\
\hline 0.96 & 791.22 & 191.22 & 0.33 & $26,770.58$ & $1,130.68$ & $27,901.26$ \\
\hline 0.97 & 795.38 & 195.38 & 0.19 & $27,352.55$ & 636.01 & $27,988.56$ \\
\hline 0.98 & 799.53 & 199.53 & 0.08 & $27,934.52$ & 282.67 & $28,217.19$ \\
\hline 0.99 & 803.69 & 203.69 & 0.02 & $28,516.48$ & 70.67 & $28,587.15$ \\
\hline 1.00 & 807.80 & 207.80 & 0.00 & $29,092.63$ & 0.01 & $29,092.64$ \\
\hline
\end{tabular}

Fuente: elaboración propia.

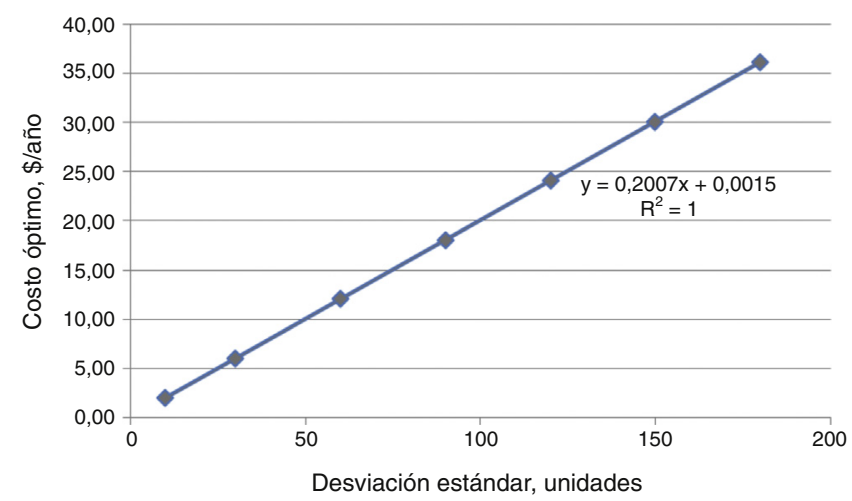

Figura 6. Cambio del costo mínimo local del inventario con $\sigma$.

Fuente: elaboración propia. 


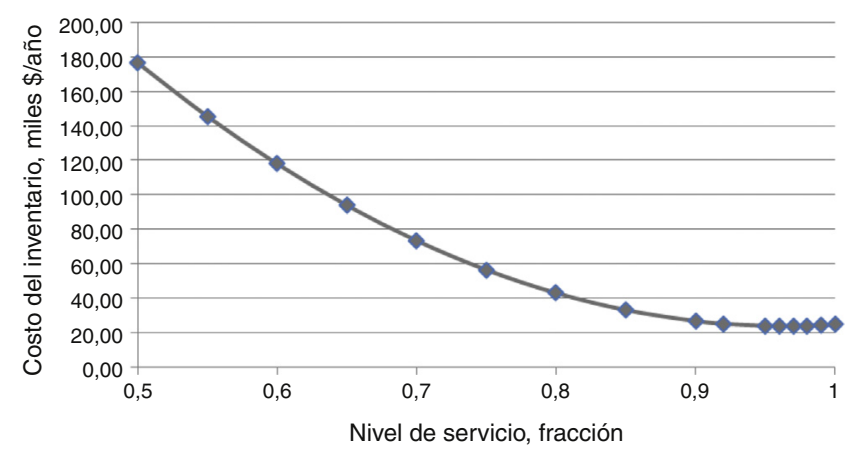

Figura 7. Variación del costo del inventario con el nivel de servicio. Fuente: elaboración propia.

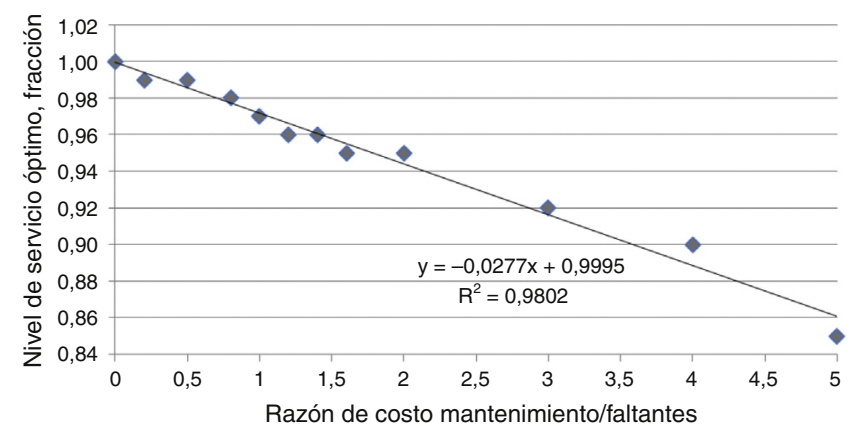

Figura 8. Cambio del nivel de servicio con la razón de costo mantenimiento/faltantes.

Fuente: elaboración propia.

Finalmente, al analizar la variación del nivel de servicio de costo mínimo con la estructura de costos, la figura 8 presenta la relación de cambio.

La relación es inversa y lineal, tal y como se esperaba, con una disminución del $2.77 \%$ en el nivel de servicio de costo mínimo por un aumento de una unidad en la razón de costos mantenimientofaltantes.

Se hicieron cambios en los costos de faltantes y mantenimiento del inventario para probar si se repetían las relaciones entre las variables bajo análisis, las cuales se mantuvieron independientemente de los distintos valores de los costos.

\section{Conclusiones}

Este estudio se ha realizado para determinar si las variaciones de la demanda de los consumidores afectan al inventario de seguridad requerido, conforme a lo señalado por Fiom (2012), lo cual ha quedado confirmado.

Con ambas distribuciones de probabilidad se han obtenido resultados similares.

Con mayor variabilidad de la demanda del tiempo de entrega, las existencias de seguridad aumentan, a razón de 1.89 unidades para el artículo con distribución normal y de 1.59 unidades para el de distribución uniforme. Esto va de acuerdo a lo que la mayoría de los académicos ha encontrado en otras investigaciones. Sin embargo, a diferencia de lo que afirma Song (1994), que 
esta relación de cambio depende de la estructura de costos, en este estudio los resultados de una relación directa entre la desviación estándar de la demanda y el inventario de seguridad se han dado para cualquier valor de la razón de costos de mantenimiento y faltantes.

De manera similar, con más variabilidad de la demanda, el costo mínimo local para ambas distribuciones aumenta, lo que está en concordancia con Song (1994). El cambio es menos pronunciado para el caso del artículo con distribución normal, comparado con el de distribución uniforme. Valdría la pena investigar con otras distribuciones diferentes, a fin de confirmar lo comentado por Dullaert y Zamparini (2013), que dependiendo de cuál sea la distribución, el costo podría aumentar o disminuir en caso de un incremento en la variabilidad de la demanda, lo que para el caso de las 2 distribuciones de probabilidad manejadas, la normal y la uniforme, no sucedió.

El nivel de servicio de costo mínimo ha sido $94.5 \%$ para el artículo con distribución normal y $96 \%$ para el de distribución uniforme, siendo un poco diferentes las curvas respectivas, ya que para el caso normal se da el mínimo en $94.5 \%$, y si el nivel de servicio sigue aumentando, el costo aumenta, mientras que en el caso del artículo con distribución uniforme se alcanza el mínimo en $96 \%$, y con niveles de servicio mayores el costo prácticamente es el mismo. Esto va de acuerdo a

Tabla 3

Comparativo de los hallazgos con las aportaciones de otros autores

\begin{tabular}{|c|c|c|}
\hline Autor(es) & Aportación & Este trabajo \\
\hline Chopra et al. & $\begin{array}{l}\text { Reducir el tiempo de entrega y su variabilidad para disminuir el } \\
\text { nivel de inventario sin caer en bajo nivel de servicio Es más } \\
\text { importante la variabilidad que la magnitud del tiempo de entrega }\end{array}$ & $\begin{array}{l}\text { OK en la primera } \\
\text { afirmación }\end{array}$ \\
\hline Fiom & $\begin{array}{l}\text { El inventario de seguridad es una protección contra la } \\
\text { incertidumbre, que incluye variaciones en la demanda de los } \\
\text { clientes y del tiempo de entrega del proveedor }\end{array}$ & $\mathrm{OK}$ \\
\hline Kanet et al. & $\begin{array}{l}\text { Ante mayor incertidumbre en el lado de la oferta y demanda de un } \\
\text { artículo, manejar un inventario de seguridad mayor }\end{array}$ & $\mathrm{OK}$ \\
\hline King & $\begin{array}{l}\text { Debe manejarse el inventario para dar el nivel de servicio meta a un } \\
\text { costo mínimo, siendo la variabilidad de la demanda del tiempo de } \\
\text { entrega la variable de mayor influencia }\end{array}$ & $\mathrm{OK}$ \\
\hline Wang y Hill & $\begin{array}{l}\text { Si el tiempo de entrega sigue una distribución gamma de } \\
\text { probabilidad, en niveles de servicio del } 60 \text { al } 70 \% \text {, si la variabilidad } \\
\text { del tiempo de entrega baja, el inventario de seguridad aumenta }\end{array}$ & No \\
\hline Nasri et al. & $\begin{array}{l}\text { Ante tiempos de entrega con distribuciones de probabilidad normal } \\
\text { y uniforme, disminuir la variabilidad del tiempo de entrega reduce } \\
\text { el costo del inventario }\end{array}$ & $\mathrm{OK}$ \\
\hline Song & $\begin{array}{l}\text { Si la variabilidad del tiempo de entrega aumenta, esto eleva la } \\
\text { demanda del tiempo de entrega y el costo del inventario, y este } \\
\text { efecto está en función de la estructura de costos del inventario en } \\
\text { los rubros de faltantes y mantenimiento }\end{array}$ & $\begin{array}{l}\text { OK en la primera, } \\
\text { No en la segunda } \\
\text { afirmación }\end{array}$ \\
\hline Dullaert y Zamparini & $\begin{array}{l}\text { El efecto de la variabilidad del tiempo de entrega en el costo del } \\
\text { inventario depende de la distribución de la demanda del tiempo de } \\
\text { entrega y el nivel de servicio meta }\end{array}$ & No \\
\hline Hayya et al. & $\begin{array}{l}\text { Para disminuir el costo del inventario hay que reducir la } \\
\text { variabilidad del tiempo de entrega }\end{array}$ & $\mathrm{OK}$ \\
\hline Fang et al. & $\begin{array}{l}\text { No es claro el efecto de reducir la varianza o la media del tiempo de } \\
\text { entrega en el costo del inventario }\end{array}$ & No \\
\hline
\end{tabular}

Fuente: elaboración propia. 
lo señalado por King (2011), de alcanzar un nivel de servicio apropiado para el cliente a un costo mínimo del inventario.

Para ambos productos se ha dado una relación inversa entre el nivel de servicio de costo mínimo y la razón de costo de mantenimiento a faltantes, lo que era de esperar, ya que si dicha razón aumenta, costará más mantener el inventario de seguridad y menos tener faltantes, lo que lleva el nivel de servicio a la baja.

En la tabla 3 se muestran de manera sintetizada varias de las aportaciones de autores incluidas en la revisión de la literatura, así como el hecho de si hay acuerdo o no con los hallazgos de este trabajo.

Puede verse que en la mayoría de los casos hay acuerdo entre las aportaciones de este estudio y las de otros autores, aunque no haya sucedido en todos los casos.

Para trabajos futuros valdría la pena investigar el caso de una demanda autocorrelacionada, ya sea positiva o negativa, a fin de contrastar lo comentado por Boute et al. (2010), que, dependiendo del signo de la correlación, será el efecto sobre las existencias de seguridad; o lo señalado por He et al. (2005) de contrastar el impacto de la media y la varianza del tiempo de entrega en el costo del inventario, para definir cuál de estas variables es más importante.

\section{Referencias}

Babai, M. Z., Syntetos, A. A., Dallery, Y. y Nikolopoulos, K. (2009). Dynamic re-order point inventory control with lead-time uncertainty: Analysis and empirical investigation. International Journal of Production Research, 47(9), 2461-2483.

Balakrishnan, A., Pangburn, M. S. y Stavrulaki, E. (2004). 'Stack Them High, Letém Fly': Lot-sizing policies when inventories stimulate demand. Management Science, 50(5), 630-644.

Barry, C. (2007). The best of inventory. Multichannel Merchant, 3(1), 1-45.

Ben-Daya, M. y Hariga, M. (2003). Lead time reduction in a stochastic inventory system with learning consideration. International Journal of Production Research, 41(3), 571-579.

Boute R.N., Disney S.M., Lambrecht M., van Houdt B. (2010). Coordinating Lead-Time and Safety Stock Decisions in a Two-Echelon Supply Chain with Autocorrelated Consumer Demand. KatholiekeUniversiteit Department of Decision Science and Information Management. Working Paper 1009: 1-29 [consultado 10 Mar 2014]. Disponible en: http://ssrn.com/abstract=1620237 o en http://dx.doi.org/10.2139/ssrn.1620237

Buzacott, J. A. y Shanthikumar, J. G. (1994). Safety stock versus safety time in MRP controlled production systems. Management Science, 40(12), 1678-1689.

Chikan, A. (2007). The new role of inventories in business: Real world changes and research consequences. International Journal of Production Economics, 108, 54-62.

Chopra, S., Reinhardt, G. y Dada, M. (2004). The effect of lead time uncertainty on safety stocks. Decision Sciences, 35(1), 1-24.

Dullaert, W. y Zamparini, L. (2013). The impact of lead time reliability in freight transport: A logistics assessment of transport economics findings. Transportation Research Part E, 49(1), 190-200.

Eppen, G. D. y Martin, R. K. (1988). Determining safety stock in the presence of stochastic lead time and demand. Management Science, 34(11), 1380-1390.

Fang, X., Zhang, C., Robb, D. J. y Blackburn, J. D. (2013). Decision support for lead time and demand variability reduction. Omega, 41(2), 390-396.

Fiom, S. G. (2012). Effective and efficient use of safety or buffer stock. Operations Management, 5, $27-31$.

Hayya, J. C., Harrison, T. P. y Chatfield, D. C. (2009). Exploring the structural properties of the (D, 0) inventory model. International Journal of Production Research, 47(10), 2767-2783.

He, X. J., Kim, J. G. y Hayya, J. C. (2005). The cost of lead-time variability: The case of the exponential distribution. International Journal of Production Economics, 97(2), 130-142.

Kanet, J. J., Gorman, M. F. y Stoblëin, M. (2010). Dynamic planned safety stocks in supply networks. International Journal of Production Research, 48(22), 6859-6880.

King, P. L. (2011). Crack the code: Understanding safety stock and mastering its equations. APICS Magazine, July/August, $33-36$. 
Kouvelis, P. y Li, J. (2008). Flexible backup supply and the management of lead-time uncertainty. Production and Operations Management, 17(2), 184-199.

Nasri, F., Affisco, J. F. y Paknejad, M. J. (1990). Setup cost reduction in an inventory model with finite-range stochastic lead times. International Journal of Production Research, 28(1), 199-212.

Ruiz-Torres, A. J. y Mahmoodi, F. (2010). Safety stock determination based on parametric lead time and demand information. International Journal of Production Research, 48(10), 2841-2857.

Silver, E. A. (1965). Bayesian determination of the reorder point of a slow moving item. Operations Research, 13(6), 989-997.

Silver, E. A. y Costa, D. (1995). Service and inventory level implications of a particular simple method of establishing the reorder point. Information Systems \& Operational Research, 33(2), 133-144.

Song, J. (1994). The effect of lead time uncertainty in a simple stochastic inventory model. Management Science, 40(5), 603-613.

Taha, H. (2004). Investigación de Operaciones (7. a ed.). México: Pearson Prentice Hall.

Van Kampen, T. J., van Donk, D. P. y van-der Zee, D. (2010). Safety stock or safety lead time: Coping with unreliability in demand and supply. International Journal of Production Research, 48(24), 7463-7481.

Wang, P. y Hill, J. A. (2006). Recursive behavior of safety stock reduction: The effect of lead-time uncertainty. Decision Sciences, 37(2), 285-290.

Wang, P., Zinn, W. y Croxton, K. L. (2010). Sizing inventory when lead time and demand are correlated. Production and operations management. 19(4), 480-484. 